\title{
Colony of Robots for Exploration Based on Multi-Agent System
}

\author{
I. Hughes, G. Millán, C. Cubillos, G. Lefranc
}

\author{
Ian Hughes, Claudio Cubillos, Gastón Lefranc* \\ Pontificia Universidad Católica de Valparaíso \\ Escuela de Ingeniería Eléctrica \\ Avda. Brasil \#2147 \\ Valparaíso - Chile \\ *Corresponding author: glefranc@ucv.cl
}

\section{Ginno Millán}

Universidad Católica del Norte

Escuela de Ingeniería

Larrondo \#1281

Coquimbo - Chile

gmillan@ucn.cl

\begin{abstract}
In this paper a colony of robots for closed environments exploration is presented. This small colony of robots, conformed by mobile robots and a quadcopter, is based on heterogeneous Multi-Agent System (MAS). The objective of the system is to quickly recognize a closed three-dimensional environment, without access to references such as a Global Positioning System (GPS), to perform exploration of each unit with different characteristics and perform a joint recognition. All communications work wirelessly with a system responsible of data collection, tracking and managing all collected information. Finally, it provides a basis for multi-agent robots which allow recognition, mapping and information gathering in places where units are efficiently deployed the entire colony's abilities.

Keywords: Colony of robots, Multi-Agent Systems (MAS), robotics.
\end{abstract}

\section{Introduction}

The distribution of tasks among different units incorporated into engineering areas is an alternative to reduce costs in contrast with one large unit to which all tasks are assigned. Distributing several tasks significantly improve performance, increase fault tolerance, among other qualities, especially when these tasks are inseparable by a single unit. In a colony of robots using multi-agent robotics, a task's distribution can have several different solutions to a variety of problems based on behaviour control by cooperation and collaboration [1], [2].

A typical task requires distribution to increase performance in spatial exploration, which has problems such as maximizing every unit and proper path planning to avoid intersections. Added problems are presented with heterogeneous units, moving capabilities, sensor type and range. Processing power increase the challenge of control due different conditions that have to be applied to each unit.

Tasks and scenarios which are assigned to the MAS will first undergo some standardization of structures and specific protocols cite3. Some of these tasks in the colony of robots are: communication, architecture, planning and control, location, map display and exploration strategies, interaction with the environment, multi-robot for air and ground coordination adaptive exploration and generation of maps [4].

The communication task in MAS has been built maintaining standardized protocols such as FIPA (Foundation for Intelligent Physical Agents) [4] that provide complete and sufficient ontological structure for virtually any application in multi-agents for both the software and mobile 
robotics. Communication with one specific robot or the group, sharing particular portions of the gathered information or simply the update rate, improves the unit or group performance [5]- [7].

Also, a control procedure is needed to verify that the global mission objectives are met and that the electronic and software architecture is capable of supporting these control mechanisms. Thus, it is possible to relegate tasks and share goals, using heterogeneous agents efficiently, capable of producing a consistent behaviour [8].

For map representation and exploration, a number of approaches and methodologies for general and specific situations are implemented. An exploration, mapping and localization are always relative to the robots and subject to inaccuracies which can be partially corrected through redundancy or loop closure algorithms [4], [7], [10].

Coordination of multiple agents has to consider each unit independently but also collectively. It has been suggested in several studies that the joint work of robots that require a high level of coordination and cooperative action, in which they must carry loads or move objects exceed the capacity of action of a single unit [4], [10].

Coordination for multi-robot exploration and mapping generation with MAS can create maps through collaborative exploration, with a technique similar to SLAM (Simultaneous Localization And Mapping) with multiple reference points added by different units and perspectives [6], [10], $[12]$.

In this paper, the implementation of a small experimental heterogeneous colony of robots is presented. This heterogeneous colony consists of mobile robots and a quadcopter, based on MAS capable of performing exploration in closed environments, to obtain 3D maps. The system executes scanning in closed environments, without access to external global references such as GPS. The recognition, mapping and information gathering takes place in the colony.

\section{The Colony of Robots}

A small colony of robots conformed by mobile robots and a quadcopter and based on a heterogeneous robotics MAS is implemented to perform exploration in closed environments, and to obtain maps. This system works by collaboratively scanning closed 3D environments to browse, navigate and communicate information using only the sensors available on each unit. To support the entire software structure, ROS (Robot Operating System) is implemented for its easy reconfiguration [11].

Figure 1 shows an overview of simple configuration of the MAS working on a bi-dimensional plane. In this case, robotic units use their sensors to identify obstacles and communicate with a central station. This communication takes place via TCP/IP messaging structure and wireless links, to share information and generate maps allowing browse previously visited places. All this is on top of a software architecture based on ROS that providing a 3D simulation platform [3]. 


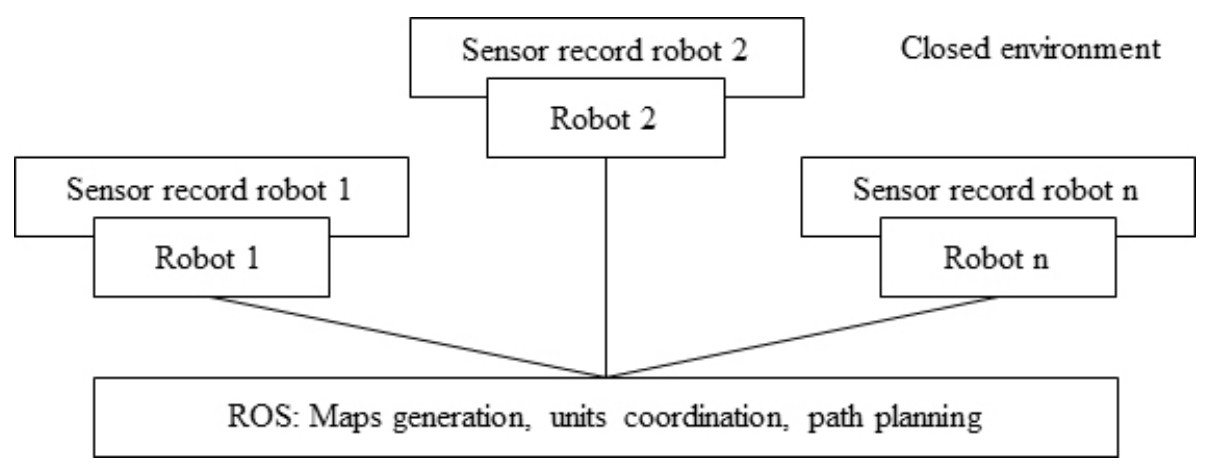

Figure 1: ROS communication with robotic units within a closed environment

This platform is capable of representing different types of sensors, robotic units and conditions before the implementation of the system results in real conditions. The simulation layers of ROS accept data from real sensors to control an output link.

The MAS easily integrates with ROS since the internal communication methods are similar to those set by FIPA. This allows complete integration for MAS to interact with ROS for specific tasks, facilitating exploration and an efficient use of the units to explore various places simultaneously while sharing information. The ROS interface show the state of the agent sensors to measure distances to tha walls, translation and rotation in all axes.

Two real units, the terrestrial mobile robots and air quadcopter (Figure 2), are used to provide real-time information displayed on ROS with respect to all sensors, inertial measurement units and distance sensors. Wireless communication is possible at approximate distance among units of $50 \mathrm{~m}$ and $150 \mathrm{~m}$ in closed environments such as the inside of a conventional house. Navigation data allow mapping environment.
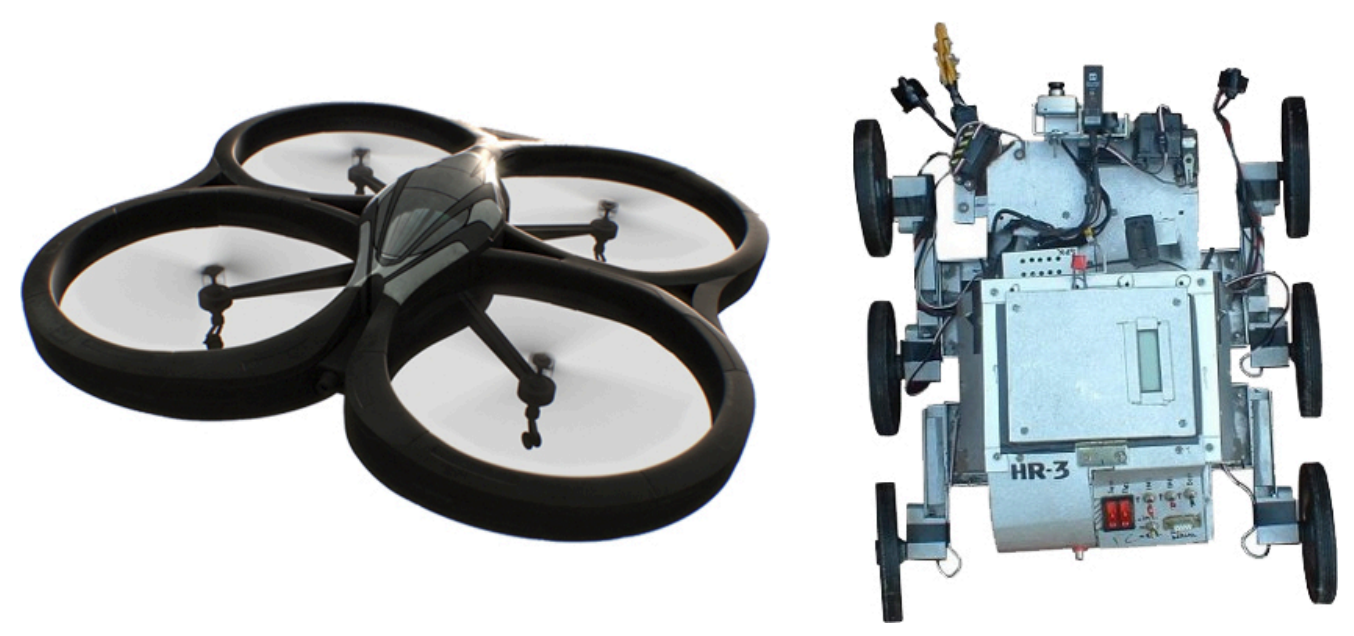

Figure 2: Robotics unit used

\section{Multi-Agent System (MAS)}

A multi-agent system is characterized by software with a robust architecture, adaptable and operates in different environments. The different objectives can be achieved in an intelligent and autonomous fashion, by exchanging information from the environment or with other agents [9]. 
A "Blackboard" type structure used as a workspace to which all agents that request access in order to share information in a bidirectional manner, considers various agent writers and readers. Each agent can see the data status, update their own, and write the task information results to make them accessible to everyone else. To have a better solution that facilitates cooperation, collaboration and communication among agents, high level moderators in charge of supervision are introduced. They monitor and evaluate the situation using the knowledge base to select the most qualified agents to solve the sub problem. A "dispatcher" is also included as responsible for informing of any new situation available on blackboards (Figure 3). Messages between among agents need to comply with a collaborative communication protocol. In this case, it complies with FIPA protocol. This protocol must specify the type of communication process and message format together with processing the semantics of agent communication language. In this case, it complies with FIPA protocol.

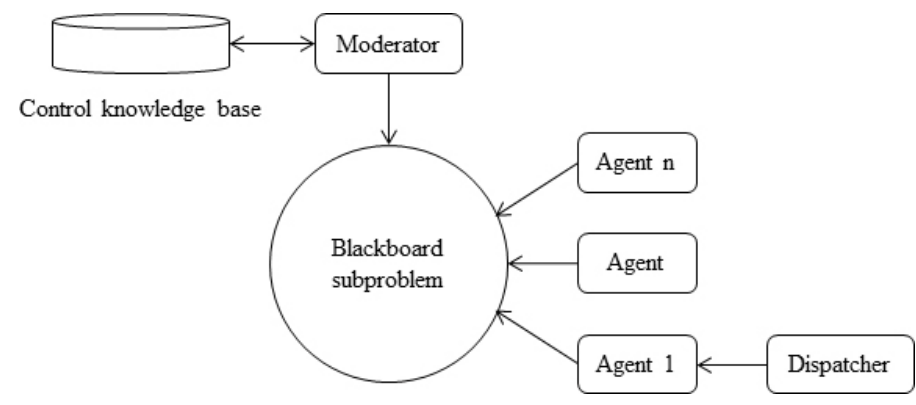

Figure 3: Model of blackboard information exchange system with modifications for optimization

\section{Multi-Agent Structure}

To carry out the proposed mission of exploration, different types of agents working collaboratively are required to carry out the task of navigating a complex environment while maintaining the integrity of the robots. The MAS structure is shown in Figure 4.

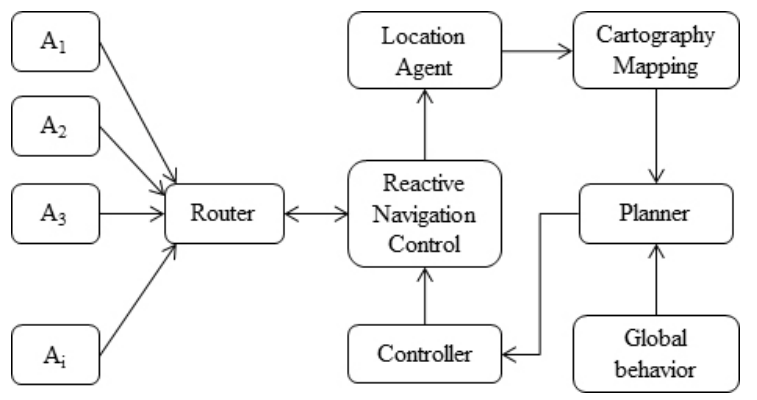

Figure 4: Control based on multi-agent for heterogeneous robot navigation

Reactive Agents: These agents are known for being the simplest and rely solely on the environment variables to give an immediate response. These reactive agents used are: Agents of perception: agents obtain environmental information from robot internal parameters (battery charge level, system status, etc.); Router Agent: it keeps track of the agents and makes the link between the control electronics and a radio transmitter to software agents; and Reactive Navigation Control Agent: this agent makes decisions to meet the requirements and requests of the highest level agents. 
Deliberative Agents: These agents are in charge of generating the maps, making control decisions and produce high-level strategies to meet the requirements of exploration, planning and control. These deliberative agents are: Planner Agent: this agent makes decisions to meet the requirements and requests of the highest level agents; Mapping Agent: it provides a system for capturing and storing navigation data collected by the sensors of the robots; Driver Agent: It flows of information from the planner agent to reactive control agent, transforming them into readable data in terms of direct control of robots; Agent interface for overall performance: This agent simply provides a query method for multi-agent system to determine the type of map to build; and Agent Location: Since the generation of maps and the location of several agents are needed localization algorithms.

\section{Evaluations}

A 2D Simulation with individual units and a test with real data, directly from the sensors of each agent, has been done. The mobile robots and the air quadcopter, provide real-time information displayed on the display of ROS with respect to the sensors and operational inertial measurement unit. In order to test a multi-agent configuration with heterogeneous units, the problem is minimized to a closed environment exploration with only a restricted amount of obstacles for the units to map. Communication between the two units (extensible to more units) establishes a framework that can be used for various types of tasks and processes for collaborative loop closure. Wireless communication allows an approximate distance between units of 150 meters and $50 \mathrm{~m}$ in closed environments, such as the inside of a house. With well-established communication, MAS travelled in a controlled fashion through the inside of a simple room. The navigation data is successfully recorded from all the incoming information and compiled into one single 3D map. For the robotic test platforms used in this research, communication is able to address every robotic unit individually, by group or by general broadcast. On top of this, the protocol using MAS strategies needs to take into account the various characteristic of each heterogeneous unit to optimize data flow.

For evaluation purposes, objects representing all possible obstacles (under the sensors minimal specifications for detectability) or errors in sensor readings are removed from the areas to be explored leaving only larger walls. Both, aerial and terrestrial units, have exactly the same capacity in sensors to generate maps and internal representations of their surroundings. After several runs all movement and rotation decisions are made according to the distance measured with three sensors, away from the nearest wall.

The ranges measured empirically with ultrasound can be displayed in a Table 1 and refer to minimum and maximum resolutions that robotic units are able to register.

Table 1: Distance and size respectively maximum and minimum detectability with the maximum opening degree sensor

\begin{tabular}{|c|c|c|}
\hline $\begin{array}{c}\text { Maximum distance to the } \\
\text { obstacle (meters) }\end{array}$ & $\begin{array}{c}\text { Minimum size obstacle } \\
\text { (meters) }\end{array}$ & $\begin{array}{c}\text { Sensor opening } \\
\text { (degrees) }\end{array}$ \\
\hline 1.8 & 0.006 & $<36$ \\
\hline 3 & 0.03 & 36 \\
\hline 3.6 & 0.083 & 40 \\
\hline$>6$ & $>0.1$ & 50 \\
\hline
\end{tabular}

The sensors arrangement, from the front and two opposite sides, has a total of 234 degrees of coverage to the front with two blind spots of 34 degrees centered at 45 degrees from the 
central axis of each robotic unit. In the case of aerial units has maximum range sonar 6 meters vertically pointing towards the ground, to keep a record high. Figure 5 shows the reading of the three sensors captured directly from a real drive and after moving interpretation.
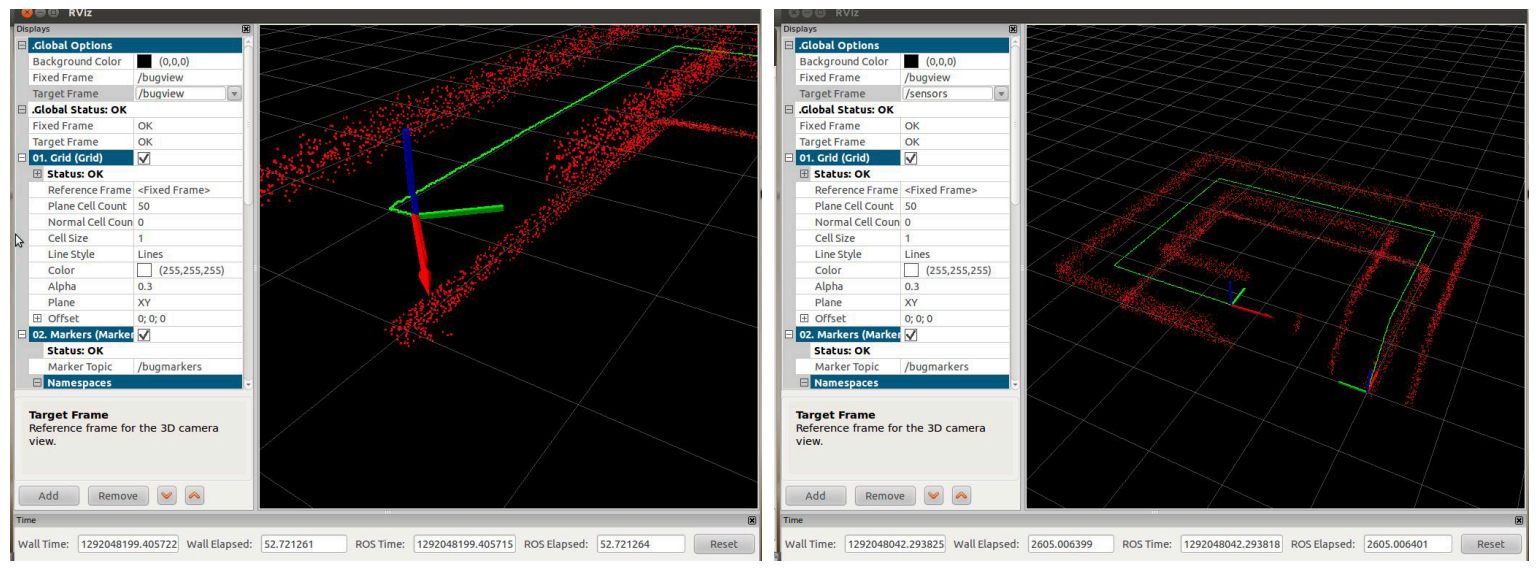

Figure 5: Data received from one of the perception agent in ROS

By removing small objects below the maximum resolution of the sensors, the environment becomes easily navigable. Obviously, the replacement of sensors by other higher resolution can also be considered as an alternative.

For the same scenario, in 10 opportunities are similar times to the end of the path indicated by the arrest of the units having made the assumption of having entire area. Since for this case are two units that perform work at different levels in a three-dimensional plane, it is assumed that a single unit would have required twice the time to explore two levels. Table 2 yields the following:

Table 2: Travel times for attempted per unit and the sum of both

\begin{tabular}{|c|c|c|c|}
\hline Try & Terrestrial time (min) & Aereal time (min) & Addition (min) \\
\hline 1 & $1: 15$ & $0: 45$ & $2: 00$ \\
\hline 2 & $1: 20$ & $0: 46$ & $2: 06$ \\
\hline 3 & $1: 16$ & $0: 47$ & $2: 04$ \\
\hline 4 & $1: 22$ & $0: 46$ & $2: 08$ \\
\hline 5 & $1: 14$ & $0: 45$ & $1: 59$ \\
\hline 6 & $1: 19$ & $0: 48$ & $2: 07$ \\
\hline 7 & $1: 16$ & $0: 44$ & $2: 00$ \\
\hline 8 & $1: 15$ & $0: 45$ & $2: 00$ \\
\hline 9 & $1: 19$ & $0: 45$ & $2: 05$ \\
\hline 10 & $1: 16$ & $0: 46$ & $2: 02$ \\
\hline
\end{tabular}

This test provides a comparison of the times it would take a single unit versus the two used in operating scheme and heterogeneous multi-agent to travel and create a map of an environment. By having multiple units, not only reduce the times, but also produces greater redundancy in the data recorded by the sensors which could lead to fewer errors, reduced costs in a single unit of sensory precision greater speed in capturing information. This is being administered by MAS, allowing the inclusion of algorithms that make searches more efficient and avoiding routes for example, visiting a point repeatedly.

During each run, the necessary data is captured to reconstruct a 3D environment and generate 
a map. In the times indicated in the above table was obtained real-time reconstruction from the data collected from the sensors, which when viewed by RVIZ of ROS, as in the simulations, can form a complete environment. In the following sequence of figures shows the map after completion of a course of 1:16 minutes of a land agent. For better representation and using the constant redundant sensor readings, each marked on the map represents average readings closest. With this you get a flatter surface and representative mapped with a maximum error of about 15 centimeters per point generated on the map.

The arrow, in Figure 6(a), represents starting point, the crosses are points of decision-making, where it alters the path avoiding obstacles. The three media circles around the starting arrow represent what a unit would be able to see at 1 meter for the inner circle, 1.5 for the next, and 2 meters for the outer circle. The sensors have a maximum range of 6 meters.

During each run, the necessary data is captured to reconstruct a three-dimensional environment and generate a map (Figure 6(d)). Times obtained in real-time reconstruction from the data collected from the sensors, takes 1:20 min to mobile robot (Figure 6(b)) and 0:46 min to quadcopter (Figure 6(c)) to get maps. In total is 2:06 min to have 3D map (Figure 5(d)), with small error.

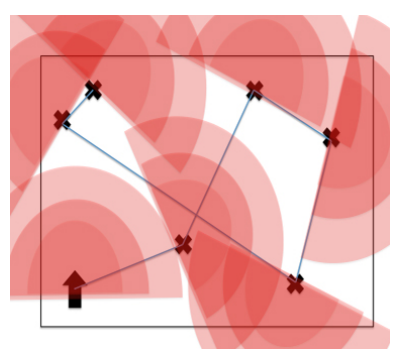

(a)

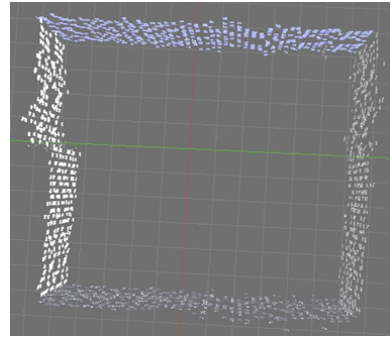

(b)

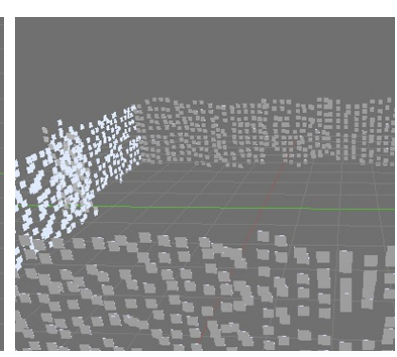

(c)

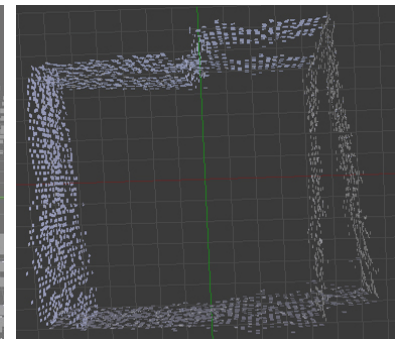

(d)

Figure 6: (a) Robots using its ultrasonic sensors, (b) Map obtained by mobile robots, (c) Map obtained by quadcopter, (d) Overlay maps generated by two units

\section{Conclusions}

This paper the implementation of a small Colony of Robots, conformed by mobile robots and quadricopter and based on heterogeneous MAS has been presented. This system is capable of performing exploration, scanning of closed three-dimensional environments, without GPS and only using collaborative techniques for global and local orientation. The system has wireless communication for data collection, track and manage all information collected. A multi-agent robot permits the recognition, mapping and information where units are deployed.

The heterogeneous MAS is capable of distributing among agents and respond appropriately, with ROS, to robot applications. MAS can control several units with different capacities and characteristics. Tests with a mobile robot and aerial unit show MAS is able to manage navigation and communicate both data and actions and commands in a scalable way.

The maps obtained match the simulated, demonstrates that communication, data transfer and displacement by mechanical agents, in real working environments, perform in accurate way with heterogeneity and cooperation among different units. 


\section{Bibliography}

[1] Lefranc, G. (2008); Colony of Robots: New Challenge, International Journal of Computers Communications \& Control, ISSN 1841-9836, 3(S):92-107.

[2] Lefranc, G. (2008); Colony of robots, in Lotfi A. Zadeh, Dan Tufis, Florin Gheorghe Filip, Ioan Dzitac (eds.), From Natural Language to Soft Computing: New Paradigms in Artificial Intelligence, Editing House of Romanian Academy, ISBN:978-973-27-1678-6.

[3] FIPA TC Architecture, FIPA Abstract Architecture; Specification, Foundation for Intelligent Physical Agents, (http://www.fipa.org), 2002.

[4] Rojas, D. et al (2013); Integration of Algorithms for Maps Construction and Simultaneous localization in a Mobile Robot. IFAC International Conference on Management and Control of Production and Logistics, Brazil, 129-134, DOI: 10.3182/20130911-3-BR-3021.00098.

[5] Roth, M.; Simmons, R.; Veloso, M. (2004); Decentralized Communication Strategies for Coordinated Multi-Agent Policies, Robotics Institute, Carnegie Mellon University.

[6] Latorre, H.; Harispe, K.; Salinas, R.; Lefranc, G. (2011); Ontology Model of a Robotics Agent Community, International Journal of Computers Communications $\&$ Control, ISSN 1841-9836, 6(1):125-133.

[7] Latorre, H.; Harispe, K.; Salinas, R.; Lefranc G. (2010); Proposed Model of Behavior for a Community of Robotic Agents, Congreso Infonor, Chile, 168-171, DOI: 10.1109/SCCC.2010.8.

[8] Pechoucek, M.; Marík, V. (2008); Industrial deployment of multi-agent technologies: review and selected case studies, Auton Agent Multi-Agent Syst, ISSN: 1387-2532, 17(3):397-431.

[9] Bermes, C. et al (2008); New Design of the Steering Mechanism for a Mini Coaxial Helicopter, IEEE Int. Conf. Intelligent Robots and Systems, Nice, France, 1236-1241, DOI: 10.1109/IROS.2008.4650769.

[10] Fredes, D.; Cubillos, C.; Lefranc, G. (2012); Mobile Robot with Multi Agent Architecture, IEEE Conference International on Engineering and Systems Applications. Chile.

[11] Quigley, M. et al (2009); ROS: an open-source Robot Operating System, In ICRA Workshop on Open Source Software.

[12] Cheein, F. A. A.; Tobeiro, J. M., di Sciascio, F.; Carelli, R.; Lobo Pereira, F. (2010); Monte Carlo Uncertainty Maps-based for Mobile Robot Autonomous SLAM Navigation, IEEE International Conference on Industrial technology, Viña del Mar, Chile, 1433-1438, DOI: 10.1109/ICIT.2010.5472495. 